\title{
Distribution Transformer Modeling for Application in Three-Phase Power Flow Algorithm
}

\author{
J. L.Choque, D. Rodas, A.Padilha-Feltrin, Senior Member
}

\begin{abstract}
In this paper, it is presented a methodology for hree-phase distribution transformer modeling, considering several types of transformer configuration, to be used in algorithms of power flow in three-phase radial distribution networks. The paper provides a detailed discussion about the models and the results from an implementation of the power flow algorithm. The results, taken from three different networks, are presented for several transformer configurations and for voltage regulators as well.
\end{abstract}

Keywords - Distribution transformer modeling, Nodal admittance matrices, Three-Phase power flow.

\section{NOMENCLATURA}

$Y_{\text {bus trafo }} \quad$ Matriz de admitância nodal do transformador;

$Y_{p p}, Y_{s s} \quad$ Submatrizes da matriz de admitância nodal $Y_{\text {bus trafo }}$ do transformador para o lado primário e secundário respectivamente;

$Y_{p s}, Y_{s p} \quad$ Submatrizes da matriz de admitância nodal $Y_{\text {bus trafo }}$ do transformador para o lado primáriosecundário e o lado secundário-primário respectivamente;

$Y I, Y I I, Y I I I \quad$ Submatrizes derivadas da $Y_{\text {bus trafo }}$;

$y_{t}$

Admitância de dispersão do transformador em p.u.;

$I_{p}^{a}, I_{p}^{b}, I_{p}^{c} \quad$ Injeção de corrente trifásica no lado primário;

$I_{s}^{a}, I_{s}^{b}, I_{s}^{c} \quad$ Injeção de corrente trifásica no lado secundário do transformador;

$V_{p}^{a}, V_{p}^{b}, V_{p}^{c}$ Tensão trifásica no lado primário do transformador;

$V_{s}^{a}, V_{s}^{b}, V_{s}^{c} \quad$ Tensão trifásica no lado secundário do transformador;

José. L. Choque é aluno de pós graduação na Unesp de Ilha Solteira, jluischoque@yahoo.es.

Dario. E. Rodas é professor da Universidad tecnologica de Pereira Colômbia,drodas@utp.edu.co.

Antonio. Padilha-Feltrin é professor da UNESP campus de Ilha Solteira, padilha@dee.feis.unesp.br.
$M$

$j n$

$I_{j}^{a}, I_{j}^{b}, I_{j}^{c}$

$S_{j n}^{a}, S_{j n}^{b}, S_{j n}^{c}$

$V_{j}^{a}, V_{j}^{b}, V_{j}^{c}$

$V_{p}^{1}, V_{p}^{2}, V_{p}^{0}$

$Y_{i}^{a}, Y_{i}^{b}, Y_{i}^{c}$

$a, b, c$

\section{INTRODUÇÃO}

$\mathrm{V}$ ários métodos de cálculo de fluxo de potência trifásico em redes radiais de distribuição surgiram nos últimos anos. Atualmente, os engenheiros de distribuidoras de energia têm disponíveis mais e melhores conjuntos de dados, de componentes da rede e de comportamento das cargas, para utilizarem em programas computacionais de cálculo de fluxo de potência. Como conseqüência, mais e melhores modelos de componentes são frequentemente necessários para serem implementados nos algoritmos de fluxo de potência e em outros.

Este artigo apresenta e discute em detalhes uma implementação de modelos de transformadores trifásico de distribuição, para uso em programas computacionais baseados no método backward / forward sweep [1], [2], [3] e [4]. Particularmente em [2] é dada ênfase à modelagem de linhas considerando-se redes a quatro fios, agora o objetivo é a consideração dos transformadores. Dentre os métodos dedicados a redes radiais (ou com poucas malhas, que são solucionados em [3]) de distribuição, este método tem se destacado pela boa característica de convergência, pouco uso de memória computacional e simplicidade de compreensão e implementação. Neste tipo de método, e em muitos outros também, surgem problemas para representação correta dos vários tipos de conexões de transformadores de distribuição.

Alguns trabalhos anteriores propuseram soluções para resolver o problema, dentre eles destacam-se as implementações [5] e [6]. Assim sugerem-se estas referências 
para encontrar uma boa revisão sobre os temas fluxo de potência trifásico e de modelagem de transformadores de rede, e também sobre as dificuldades de modelagem devido a problemas como singularidade de matrizes. Cabe destacar que algumas publicações clássicas na área, dentre elas [7], [8] e [9], não detalham como implementar modelos diferentes do Yg-Yg em programas de fluxo de potência trifásico.

Neste trabalho segue-se a linha de modelagem discutida em [5] e [6], procurando-se detalhar e discutir as implementações de forma a dar uma contribuição adicional na implementação de modelos de transformadores trifásicos.

\section{ModelaGEM DE TRANSFORMADORES DE DiSTRIBUIÇÃO}

Os transformadores trifásicos podem ser representados partindo-se da matriz admitância primitiva. Considerando esta primitiva pode-se obter a matriz admitância nodal para as diversas conexões, conforme descrito em [7], [8] e [9]. Por facilidade de representação das diferentes conexões, pode-se colocar a matriz admitância nodal na forma de submatrizes (3x3) conforme a equação (1).

$$
Y_{\text {bus trafo }}=\left[\begin{array}{c:c}
Y_{\mathrm{pp}} & \mathrm{Y}_{\mathrm{ps}} \\
\hdashline \mathrm{Y}_{\mathrm{sp}} & \mathrm{Y}_{\mathrm{ss}}
\end{array}\right]
$$

O arranjo das submatrizes de $Y_{b u s}$ do transformador permite representar as diferentes conexões dos transformadores de distribuição. Nas Tabelas 1 e 2 são mostradas as submatrizes correspondentes aos tipos mais comuns de conexões, tanto para transformadores abaixadores (Tabela 1) quanto transformadores elevadores (Tabela 2). Estas tabelas podem ser comparadas com as apresentadas em [1], [6], [10], [11] e [12]. Para ilustrar como são obtidas estas tabelas, no Apêndice A mostra-se o caso da obtenção da $Y_{\text {bus trafo }}$ para as conexões $\mathrm{Yg}-\mathrm{Yg}$ e D - Yg para transformador elevador, incluindo taps de ambos os lados.

TABELA I

SUBMATRIZES PARA CONEXÕES DO TRANSFORMADOR ABAIXADOR

\begin{tabular}{|c|c|c|c|c|c|}
\hline \multicolumn{2}{|c|}{ Conexão } & \multicolumn{2}{|c|}{ Admitância Própria } & \multicolumn{2}{|c|}{ Admitância Mútua } \\
\hline Primário & Secundário & $\mathbf{Y}_{\mathrm{pp}}$ & $\mathbf{Y}_{\mathrm{ss}}$ & $\mathbf{Y}_{\mathrm{ps}}$ & $\mathbf{Y}_{\mathrm{sp}}$ \\
\hline $\mathrm{Yg}$ & $\mathrm{Yg}$ & YI & YI & $-\mathrm{YI}$ & -YI \\
\hline $\mathrm{Yg}$ & $\mathrm{Y}$ & YII & YII & -YII & -YII \\
\hline $\mathrm{Yg}$ & $\Delta$ & YI & YII & YIII & YIII $^{\mathrm{T}}$ \\
\hline $\mathrm{Y}$ & $\mathrm{Yg}$ & YII & YII & -YII & -YII \\
\hline $\mathrm{Y}$ & $\mathrm{Y}$ & YII & YII & -YII & -YII \\
\hline $\mathrm{Y}$ & $\Delta$ & YII & YII & YIII & $\mathrm{YIII}^{\mathrm{T}}$ \\
\hline$\Delta$ & $\mathrm{Yg}$ & YII & YI & YIII & YIII $^{\mathrm{T}}$ \\
\hline$\Delta$ & $\mathrm{Y}$ & YII & YII & YIII & YIII $^{\mathrm{T}}$ \\
\hline$\Delta$ & $\Delta$ & YII & YII & -YII & -YII \\
\hline
\end{tabular}

TABELA II

SUBMATRIZES PARA CONEXÕES DO TRANSFORMADOR ELEVADOR

\begin{tabular}{|c|c|c|c|c|c|}
\hline \multicolumn{2}{|c|}{ Conexão } & \multicolumn{2}{|c|}{ Admitância Própria } & \multicolumn{2}{|c|}{ Admitância Mútua } \\
\hline Primário & Secundário & $\mathbf{Y}_{\mathrm{pp}}$ & $\mathbf{Y}_{\mathrm{ss}}$ & $\mathbf{Y}_{\mathrm{ps}}$ & $\mathbf{Y}_{\mathrm{sp}}$ \\
\hline Yg & $\mathrm{Yg}$ & YI & YI & $-\mathrm{YI}$ & $-\mathrm{YI}$ \\
\hline $\mathrm{Yg}$ & $\mathrm{Y}$ & YII & YII & -YII & -YII \\
\hline $\mathrm{Yg}$ & $\Delta$ & YI & YII & YIII $^{\mathrm{T}}$ & YIII \\
\hline $\mathrm{Y}$ & Yg & YII & YII & -YII & -YII \\
\hline $\mathrm{Y}$ & $\mathrm{Y}$ & YII & YII & -YII & -YII \\
\hline $\mathrm{Y}$ & $\Delta$ & YII & YII & $\mathrm{YIII}^{\mathrm{T}}$ & YIII \\
\hline$\Delta$ & $\mathrm{Yg}$ & YII & YI & $\mathrm{YIII}^{\mathrm{T}}$ & YIII \\
\hline$\Delta$ & $\mathrm{Y}$ & YII & YII & YIIII $^{\mathrm{T}}$ & YIII \\
\hline$\Delta$ & $\Delta$ & YII & YII & -YII & -YII \\
\hline
\end{tabular}

Em que:

$$
\begin{aligned}
& \mathrm{YI}=\left[\begin{array}{ccc}
1 & 0 & 0 \\
0 & 1 & 0 \\
0 & 0 & 1
\end{array}\right] \cdot \mathrm{y}_{\mathrm{t}} \\
& \mathrm{YII}=\frac{1}{3}\left[\begin{array}{ccc}
2 & -1 & -1 \\
-1 & 2 & -1 \\
-1 & -1 & 2
\end{array}\right] \cdot \mathrm{y}_{\mathrm{t}} \\
& \mathrm{YIII}=\frac{1}{\sqrt{3}}\left[\begin{array}{ccc}
-1 & 1 & 0 \\
0 & -1 & 1 \\
1 & 0 & -1
\end{array}\right] \cdot \mathrm{y}_{\mathrm{t}}
\end{aligned}
$$

Podem-se relacionar correntes e tensões primárias e secundárias do transformador (para fins de cálculo de fluxo de potência) com uso da matriz da equação (1):

$$
\left[\begin{array}{l}
\mathrm{I}_{\mathrm{p}} \\
\mathrm{I}_{\mathrm{s}}
\end{array}\right]=\left[\begin{array}{c:c}
\mathrm{y}_{\mathrm{pp}} & \mathrm{y}_{\mathrm{ps}} \\
\hdashline \mathrm{y}_{\mathrm{sp}} & \mathrm{y}_{\mathrm{ss}}
\end{array}\right] \cdot\left[\begin{array}{c}
\mathrm{V}_{\mathrm{p}} \\
\mathrm{V}_{\mathrm{s}}
\end{array}\right]
$$

\section{REPRESENTAÇÃO DOS TRANSFORMADORES NO AlgoritMo DE FluXo de PotênCIA BACKWARd / ForWARd SWEEP}

As tradicionais implementações do algoritmo Backward / Forward [3], [5] e [13] para o fluxo de potência necessitam basicamente, obter:

- Etapa Backward - Cálculo das injeções de correntes em todas as barras devido a cargas diretamente conectadas, assim como, cálculo dos fluxos de correntes em todas as linhas, usando-se os valores de tensão estimados (primeira iteração) ou calculados (demais iterações). Nesta etapa os cálculos são realizados partindo-se dos extremos do alimentador e seguindo-se para a subestação.

- Etapa Forward - Cálculo das tensões em todos os nós, utilizando-se os valores de fluxo de correntes obtidos na etapa backward. Nesta etapa parte-se da subestação (nó fonte) e segue-se para os nós extremos.

Na Fig. 1 destaca-se uma seção de linha de uma rede radial, na qual foi incluído o transformador de distribuição. A partir 
desta figura serão detalhados os cálculos necessários nas etapas Backward e Forward (entre duas barras conectadas por um transformador).

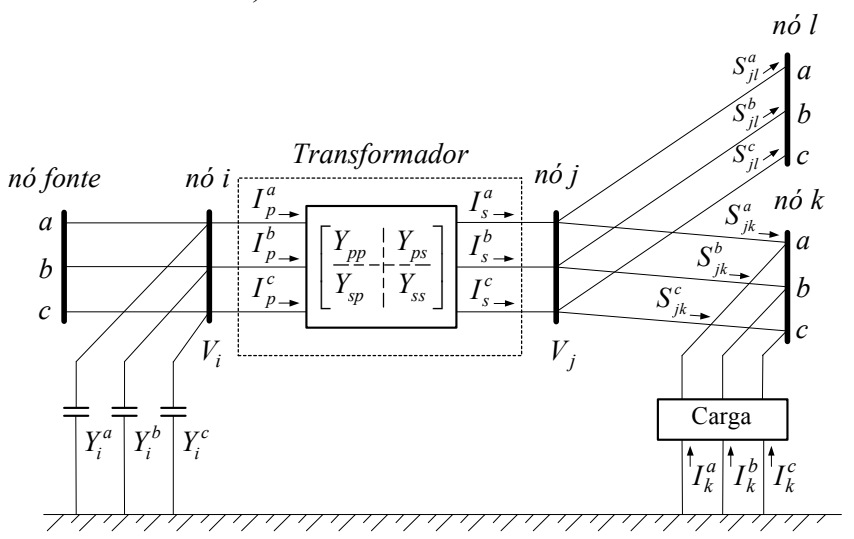

Figura 1. Seção de linha trifásica com o transformador embutido.

\section{A. Etapa Backward}

1) Injeção de Corrente na Barra Terminal do Secundário Se o secundário do transformador está conectado diretamente na barra “ $j$ ”, como mostrado na Fig. 1, pode-se obter a corrente no secundário a partir da diferença entre as correntes que entram e que saem do nó “ $j$ ” ( $I_{j}$ representa a contribuição de cargas, se houver, conectadas diretamente na barra). Assim, tem-se:

$$
I_{s}=I_{j}-\sum_{j n \in M}\left[\begin{array}{l}
\left(S_{j n}^{a} / V_{j}^{a}\right)^{*} \\
\left(S_{j n}^{b} / V_{j}^{b}\right)^{*} \\
\left(S_{j n}^{c} / V_{j}^{c}\right)^{*}
\end{array}\right]
$$

\section{2) Injeção de Corrente na Barra Terminal do Primário}

No procedimento backward sweep $V_{s}$ e $I_{s}$ são conhecidos, enquanto que $V_{p}$ e $I_{p}$ são os cálculados.

A corrente no terminal primário não pode ser obtida de forma similar àquela que foi utilizada para a corrente no secundário, porém deve-se usar a equação (5), ou mais especificamente:

$$
I_{p}=Y_{p p} V_{p}+Y_{p s} V_{s}
$$

Mas a tensão do lado primário $V_{p}$ a ser utilizada não pode ser estimada, e sim calculada de forma a levar para o lado primário o efeito da injeção de corrente existente no secundário. Assim, embora tradicionalmente não existam cálculos de tensão na etapa Backward, agora eles são necessários para ser possível considerar corretamente os modelos dos transformadores. Portanto, conhece-se a tensão $V_{s}$ (estimada para a primeira iteração ou calculada para as seguintes iterações na etapa Forward) e a corrente do secundário $I_{s}$ (calculada no passo anterior), enquanto que a tensão $V_{p}$ e a corrente $I_{p}$ do primário devem ser calculadas. A corrente do primário $I_{p}$ pode ser obtida a partir da equação (7), desde que antes seja calculada a tensão $V_{p}$. Esta tensão deve ser obtida a partir da equação (5), ou seja:

$$
V_{p}=Y_{s p}^{-1}\left(I_{s}-Y_{s s} V_{s}\right)
$$

É importante notar que a submatriz $Y_{s p}$ é singular para todas as conexões dos transformadores, exceto para $\mathrm{Yg}$-Yg. Isto é observado melhor através das equações (2), (3) e (4), onde se pode ver que ambas submatrizes $Y I I$ e $Y I I I$ são singulares, e $Y I$ é não singular. Em essência, a singularidade das matrizes dessas configurações de transformadores aparece devido a falta de um ponto de referência de tensão para ambos os lados do transformador.

Para poder representar todos os tipos de conexões de transformadores no algoritmo Backward / Forward é preciso evitar o problema da singularidade da $Y_{s p}$ na etapa Backward.

\section{a) Resolvendo o problema de Singularidade na Etapa Backward}

A fim de resolver o problema de tal singularidade, faz-se a transformação da tensão do primário $V_{p}$ em suas componentes simétricas.

$$
V_{p}=V_{p}^{(1+2)}+V_{p}^{0}
$$

Em que $V_{p}^{(1+2)}$ representa as componentes de seqüência positiva e negativa, e $V_{p}^{0}$ a componente de seqüência zero.

Assim pode-se re-escrever a equação (8) como:

$$
Y_{s p}\left(V_{p}^{(1+2)}+V_{p}^{0}\right)=I_{s}-Y_{s s} V_{s}
$$

O produto de $Y_{s p}$ por $V_{p}^{0}$ é sempre zero para todas as configurações nas quais $Y_{s p}$ é representada por $Y I I$ ou $Y I I I$ (ou ainda $Y I I I^{t}$ ), portanto:

$$
\begin{aligned}
& Y I I . V_{p}^{0}=0 \\
& \text { YIII } . V_{p}^{0}=0 \quad \text { e } \quad Y I I I^{t} \cdot V_{p}^{0}=0
\end{aligned}
$$

Então, a equação (10), exceto para a conexão $\mathrm{Yg}-\mathrm{Yg}$, pode ser reduzida a:

$$
Y_{s p} V_{p}^{(1+2)}=I_{s}-Y_{s s} V_{s}
$$


A equação (13) indica que a componente de seqüência zero de $V_{p}$ não afeta o cálculo na etapa Backward para transformadores com uma matriz $Y_{s p}$ singular. A análise acima mostra que a equação (13) pode ser usada para calcular o vetor $V_{p}$ e seus componentes de seqüência não zero $V_{p}^{(1+2)}$. Então, desde que $V_{s}^{(1+2)}$ não contem a componente de seqüência zero, pode-se escrever:

$$
\left[\begin{array}{lll}
1 & 1 & 1
\end{array}\right] \cdot V_{p}^{(1+2)}=0
$$
em:

As equações (13) e (14) podem ser combinadas, resultando

$$
Y_{s p}^{r} V_{p}^{(1+2)}=I_{s}^{r}-Y_{s s}^{r} V_{s}
$$

Em que: $Y_{s p}^{r}$ é obtida pela substituição da última linha de $Y_{s p}$ com [lll 11 , enquanto que $I_{s}^{r}$ e $Y_{s s}^{r}$ são iguais a $I_{s}$ e $Y_{s s}$ (com as últimas linhas iguais a zero), respectivamente.

Com uso da equação (15), calculam-se as componentes de seqüência não zero das tensões das duas fases do lado primário, ou seja:

$$
V_{p}^{r}=Y_{s p}^{r-1}\left(I_{s}^{r}-Y_{s s}^{r} V_{s}\right)
$$

\section{3) Cálculo das Injeções de Potência:}

A injeção de potência sobre o lado primário pode ser calculada como em [5] e [11] por:

$$
S_{p}=V_{p} I_{p}^{*}
$$

Em que:

$$
I_{p}=Y_{p p} V_{p}+Y_{p s} V_{s}
$$

Note-se que a $I_{p}$ acima, pode ser obtida da equação (5), a qual pode ser função das tensões de ambos os lados do transformador.

\section{B. Etapa Forward}

1) Cálculo da Tensão do Secundário:

O cálculo da tensão no lado secundário do transformador é realizado com uso da equação (19), deduzida de (5):

$$
V_{s}=Y_{s s}^{-1}\left(I_{s}-Y_{s p} V_{p}\right)
$$

Note-se que em (19) pode haver problemas, tendo em vista que $Y_{s s}$ pode ser uma matriz singular, dependendo da conexão do transformador. A submatriz $Y_{s s}$ tem inversa somente para as conexões Yg-Yg e D-Yg. Para a conexão YgYg, as equações (7), (8) e (19) podem ser diretamente usadas para o cálculo das etapas backward / forward. Para a configuração D-Yg somente a (19) pode ser usada na etapa forward. Para todos os demais tipos de conexões aparece a singularidade de matrizes, devido a falta de um ponto de referência de tensão em um ou em ambos os lados do transformador.

\section{a) Resolvendo o Problema de Singularidade na Etapa Forward}

Para evitar o problema de singularidade de matrizes, as componentes de seqüência não zero das tensões podem ser determinadas. Assim, pode-se re-escrever (19) como:

$$
Y_{s s} V_{s}=I_{s}-Y_{s p} V_{p}
$$

Sabendo-se que $V_{s}^{(1+2)}$ representa as componentes de seqüência positiva e negativa, isto é:

$$
V_{s}^{(1+2)}=V_{s}-V_{s}^{0}
$$

Onde o vetor $V_{s}^{0}$ é tensão de seqüência zero sobre o lado secundário. Desta forma, substituindo-se a equação (21) em (20), obtém-se:

$$
Y_{s s}\left(V_{s}^{(1+2)}+V_{s}^{0}\right)=I_{s}-Y_{s p} V_{p}
$$

O produto de $Y_{s s}$ por $V_{s}^{0}$ é sempre zero para todas as configurações de transformadores, exceto para as configurações $\mathrm{Yg}-\mathrm{Yg}$ e D-Yg. Isto acontece porque $Y_{s s}$ é representado por YII em todas as outras configurações, portanto, de (11) e (12), pode-se obter:

$$
Y I I . V_{s}^{0}=0
$$

Então a equação (22), exceto para as conexões Yg - Yg e D - Yg, pode ser reduzida a:

$$
Y_{s s} V_{s}^{(1+2)}=I_{s}-Y_{s p} V_{p}
$$

A equação (24) indica que a componente de seqüência zero de $V_{s}$ não afeta o cálculo na etapa forward para transformadores com uma matriz $Y_{s s}$ singular. A análise acima mostra que a equação (19) pode ser usada para calcular ambos: o vetor $V_{s}$, e suas componentes de seqüência não zero $V_{s}^{(1+2)}$. Então, desde que $V_{s}^{(1+2)}$ não contem a componente de 
seqüência zero, pode-se escrever:

$$
\left[\begin{array}{lll}
1 & 1 & 1
\end{array}\right] \cdot V_{s}^{(1+2)}=0
$$

As equações (24) e (25), podem ser combinadas como:

$$
Y_{s s}^{w} V_{s}^{(1+2)}=I_{s}^{w}-Y_{s p}^{w} V_{p}
$$

Em que: $V_{s}^{(1+2)}$ é a componente de seqüência não zero de $V_{s}$; a submatriz $Y_{s s}^{w}$ é a mesma submatriz $Y_{s s}$, exceto que a última linha é substituída com $\left[\begin{array}{lll}1 & 1 & 1\end{array}\right]$; o vetor $I_{s}^{w}$ e a submatriz $Y_{s p}^{w}$ são obtidas pelo substituição dos elementos na última linha de $I_{s}$ e $Y_{s p}$ por zero, respectivamente.

Uma vez que as componentes de seqüência não zero de $V_{p}$ e $V_{s}$ são calculados, a componente de seqüência zero é agregada a eles, para formar a tensão linha - neutro, tal que o procedimento backward / forward possa continuar.

\section{Testes e Resultados}

Nesta seção, apresentam-se alguns testes e os resultados obtidos com três sistemas de distribuição.

\section{A. Sistema de 2 barras}

Neste caso, tem-se um sistema de duas barras [5] e [10], como mostrado na Fig. 2, que são ligadas com um transformador trifásico abaixador em conexão delta - estrela aterrada (D-Yg). A carga no secundário do transformador é de $400+\mathrm{j} 300 \mathrm{kVA}$. Assume-se que o transformador está operando em condições nominais, portanto os taps do primário e do secundário são iguais à unidade. A tensão na barra 1 é assumida como sendo 1.0 p.u., a carga é desbalanceada: $50 \%$ para a fase a; $30 \%$ para a fase b; e $20 \%$ para a fase c. Os dados nominais do transformador são: tensões de $13,8 \mathrm{kV}-208 \mathrm{kV}$; potência de $1000 \mathrm{kVA} ; \mathrm{Z}=6 \%$; e $\mathrm{X} / \mathrm{R}=5$.

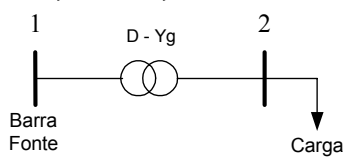

Figura 2. Sistema exemplo de dois barras

Na Tabela III apresenta-se o perfil de tensão na barra de carga, estando a carga conectada em estrela. A tolerância para a convergência foi de 0,01 . As magnitudes das tensões $|\mathrm{V}|$ estão em p.u., e os ângulos “ $\theta$ ” de cada fase estão em graus. Os valores mostrados na Tabela 3 são os de cada iteração até a convergência.
TABELA III

PERFIL DE TENSÃo DO SISTEMA DE DUAS BARRAS

\begin{tabular}{|c|c|c|c|c|c|c|}
\hline \multirow{2}{*}{$\begin{array}{c}\text { Iterac. } \\
\text { No. }\end{array}$} & \multicolumn{2}{|c|}{ Fase a } & \multicolumn{2}{c|}{ Fase b } & \multicolumn{2}{c|}{ Fase c } \\
\cline { 2 - 7 } & $\left.\mathbf{|}\right|^{\mathbf{a}}$ & $\boldsymbol{\theta}$ & $|\mathbf{V}|$ & $\boldsymbol{\theta}$ & $\mathbf{V} \mid$ & $\boldsymbol{\theta}$ \\
\hline 0 & 1,0 & 0,0 & 1,0 & $-120,0$ & 1,0 & 120,0 \\
\hline 1 & 0,9572 & 28,27 & 0,9819 & $-91,51$ & 0,9867 & 148,27 \\
\hline 2 & 0,9648 & 27,95 & 0,9825 & $-90,91$ & 0,9934 & 149,11 \\
\hline 3 & 0,9645 & 28,23 & 0,9794 & $-91,06$ & 0,9861 & 149,30 \\
\hline
\end{tabular}

\section{B. Sistema IEEE 4 barras}

Na Fig. 3 mostra-se o sistema IEEE- 4 barras [14]. O principal propósito de usar este alimentador é para proporcionar diversos testes com várias conexões de transformadores trifásicos abaixadores, bem como elevadores. Os casos testados para este alimentador incluem as seguintes conexões de transformadores: (Estrela aterrado - Estrela aterrado (Yg-Yg); Estrela aterrado - Delta (Yg-D); Estrela Delta (Y-D); Delta - Estrela aterrado (D-Yg); Delta - Delta (D-D); Estrela aberto - Delta aberto (Yo-Do)). A carga pode ser balanceada bem como desbalanceada.

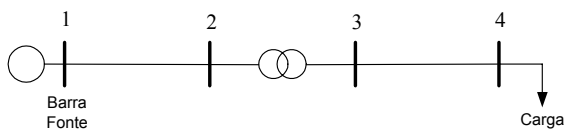

Figura 3. Sistema IEEE-4 barras

$\mathrm{Na}$ Tabela 4 são apresentados os valores de tensão trifásica para cada fase (a, b e c) na barra de carga (barra 4), para as várias configurações do transformador elevador entre as barras (2-3), sendo a carga balanceada, conectada em estrela e modelada como potência constante. A tensão é elevada de $12,47 \mathrm{kV}$ (barra 2) para 24,9 kV (barra 3). A tolerância utilizada foi de $10^{-3}$, estando os taps tanto no primário como no secundário iguais a unidade, e aplicando-se uma tensão inicial de 1,0 p.u. na barra de referência.

TABELA IV

PERFIL DE TENSÃo do Sistema IEEE-4 BARRAS

\begin{tabular}{|c|c|c|c|c|c|c|c|}
\hline Conexão & Iterações & \multicolumn{2}{|c|}{ Fase a } & \multicolumn{2}{c|}{ Fase b } & \multicolumn{2}{c|}{ Fase c } \\
\cline { 3 - 8 } Trafo & No. & $|\mathbf{V}|$ & $\boldsymbol{\theta}$ & $|\mathbf{V}|$ & $\boldsymbol{\theta}$ & $|\mathbf{V}|$ & $\boldsymbol{\theta}$ \\
\hline $\mathrm{Yg}-\mathrm{Yg}$ & 3 & 0,9489 & $-3,4711$ & 0,9524 & $-123,4870$ & 0,9510 & 116,4404 \\
\hline $\mathrm{Yg}-\mathrm{D}$ & 4 & 0,9469 & $-33,2231$ & 0,9495 & $-153,1654$ & 0,9492 & 86,6907 \\
\hline $\mathrm{Y}-\mathrm{D}$ & 4 & 0,9576 & 26,7543 & 0,9592 & $-93,3034$ & 0,9578 & 146,6640 \\
\hline $\mathrm{D}-\mathrm{Yg}$ & 4 & 0,9541 & 26,3908 & 0,9567 & $-93,6639$ & 0,9545 & 146,2547 \\
\hline $\mathrm{D}-\mathrm{D}$ & 3 & 0,9493 & $-3,4688$ & 0,9522 & $-123,4660$ & 0,9510 & 116,4071 \\
\hline Yo - Do & 4 & 0,9751 & $-30,0690$ & 0,9887 & $-150,1749$ & 0,9804 & 89,2181 \\
\hline
\end{tabular}

\section{Sistema IEEE 34 barras}

Na Fig.4 mostra-se o diagrama unifilar do sistema IEEE34 barras, como descrito como em [13]. Na figura observa-se um transformador abaixador no trecho 832-888. O trecho 888890 é um trecho longo e responsável por $25 \%$ do carregamento do sistema. 


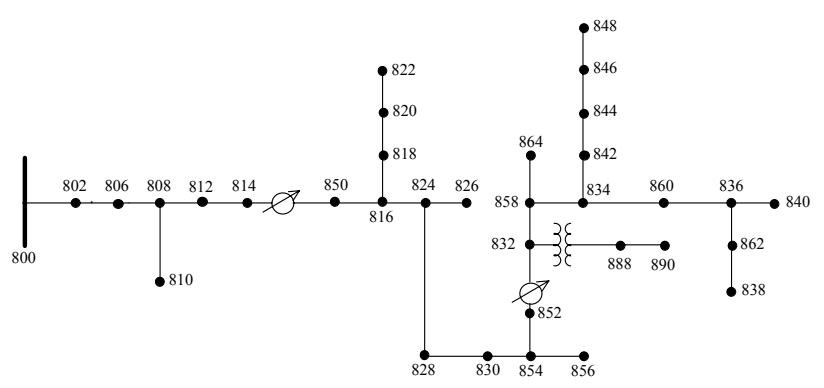

Figura 4. Sistema IEEE-34 barras.

Na Fig. 5 é apresentado o perfil de tensão do sistema IEEE34 barras, para a configuração do transformador trifásico abaixador em estrela aterrado - estrela aterrado ( $\mathrm{Yg}-\mathrm{Yg}$ ), que abaixa a tensão de $24,9 \mathrm{kV}$ para $4,16 \mathrm{kV}$. A barra terminal (890) apresenta um péssimo perfil de tensão, como pode ser visto na Fig. 5, com as cargas do sistema desbalanceadas em cada fase $(a, b$ e $c)$. Os resultados mostrados neste gráfico foram obtidos sem consideração dos dois reguladores de tensão trifásicos. Na subestação (barra 800) a tensão inicial foi de 1,05 p.u. (barra de referência). A tolerância usada para a convergência foi de $10^{-4}$. O número de iterações obtidas para alcançar a convergência foi igual a 7. Observa-se também na Fig.5, que a curva desenhada foi somente para as barras $(800$, $802,806,808,812,814,850,816,824,828,830,854,852$, 832,888 e 890 ), apenas para ilustrar as tensões nessas barras do ramal principal.

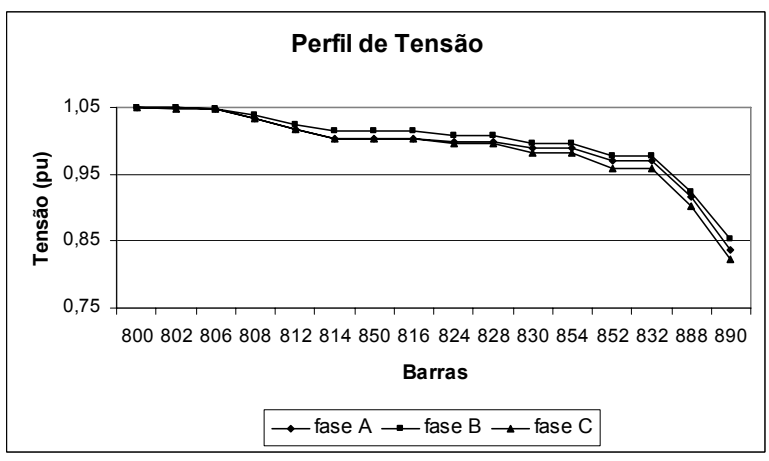

Figura 5. Perfil de Tensão do sistema sem os reguladores.

Na Fig. 6 é apresentado o perfil de tensão do sistema IEEE34 barras, com a configuração do transformador trifásico abaixador em estrela aterrado - estrela aterrado ( $\mathrm{Yg}-\mathrm{Yg})$, com as mesmas condições anteriores. Neste caso aplicaram-se os dois reguladores de tensão encontrados entre as barras (814 $850)$, e entre as barras (852-832). O número de iterações obtidas para alcançar a convergência foi de 18 . Neste caso pode-se verificar que a tensão na barra (890) melhorou devido aos reguladores, atingindo um nível de tensão razoável.

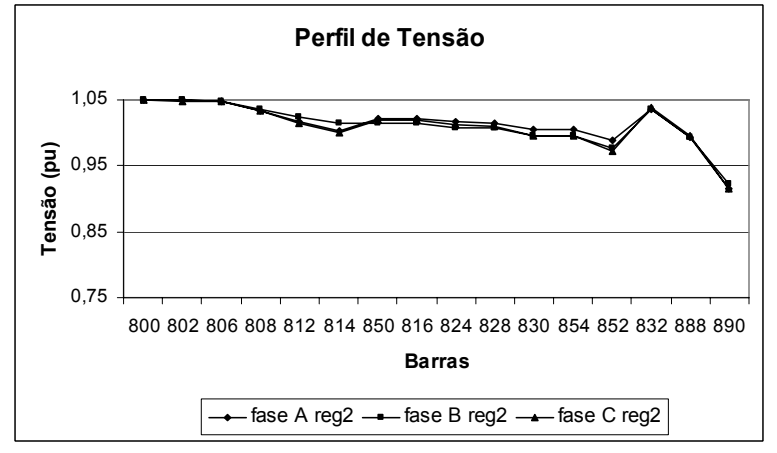

Figura 6. Perfil de Tensão do sistema com a atuação dos dois reguladores.

\section{CONCLUSÕES}

Neste trabalho foi proposta uma metodologia para modelar matematicamente os transformadores trifásicos de distribuição, considerando suas diversas configurações como abaixador ou elevador, para aplicação em fluxo de potência trifásico para sistemas de distribuição radial.

A metodologia proposta apresenta uma formulação em valores em p.u., para representar o transformador trifásico e suas diferentes configurações encontradas dentro de um sistema de energia.

Apresentaram-se detalhadamente as tabelas para as conexões do transformador abaixador bem como elevador, fazendo uso das três submatrizes $Y I, Y I I$ e $Y I I I$ (derivadas da $\left.Y_{\text {bus trafo }}\right)$ para montar a conexão desejada, nas quais pode-se levar em conta a defasagem angular e a incidência de taps.

Um dos problemas da aplicação dos modelos de transformadores em fluxo de potência é a singularidade de matrizes. Neste trabalho foi mostrado, com detalhes, quando isto ocorre e uma forma eficiente de resolver este problema. Finalmente foram apresentados resultados de cálculo de fluxo de potência, que foram obtidos considerando a modelagem apresentada.

\section{APÊNDICE A: Transformador Estrela aterrado - Estrela aterrado.}

A Fig.A1 mostra o modelo do circuito acoplado do transformador estrela aterrado - estrela aterrada.

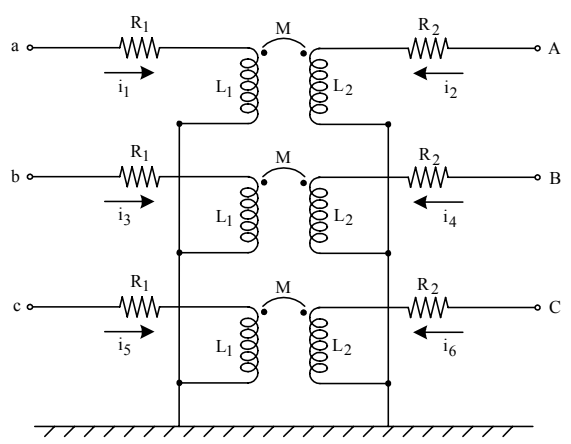

Figura A.1. Modelo do circuito acoplado do transformador estrela aterrado estrela aterrada (Yg-Yg). 
A matriz $\left[Y_{\text {bus trafo }}\right]$ do transformador mostrada na Tabela 1, para este conexão é detalhada a seguir, onde as quatro submatrizes são não singulares. Os taps do lado primário do transformador " $\alpha$ " e os taps do lado secundário " $\beta$ " podem ser diferentes da unidade.

$$
Y_{\text {bus trafo }}=\left[\begin{array}{ccc:ccc}
\frac{y_{t}}{\alpha^{2}} & 0 & 0 & -\frac{y_{t}}{\alpha \beta} & 0 & 0 \\
0 & \frac{y_{t}}{\alpha^{2}} & 0 & 0 & -\frac{y_{t}}{\alpha \beta} & 0 \\
0 & 0 & \frac{y_{t}}{\alpha^{2}} & 0 & 0 & -\frac{y_{t}}{\alpha \beta} \\
\hdashline-\frac{y_{t}}{\alpha \beta} & 0 & 0 & \frac{y_{t}}{\beta^{2}} & 0 & 0 \\
0 & -\frac{y_{t}}{\alpha \beta} & 0 & 0 & \frac{y_{t}}{\beta^{2}} & 0 \\
0 & 0 & -\frac{y_{t}}{\alpha \beta} & 0 & 0 & \frac{y_{t}}{\beta^{2}}
\end{array}\right]
$$

\section{APÊNDICE B: Transformador Delta - Estrela aterrado.}

A Fig.B1 mostra o modelo do circuito acoplado do transformador delta - estrela aterrada.

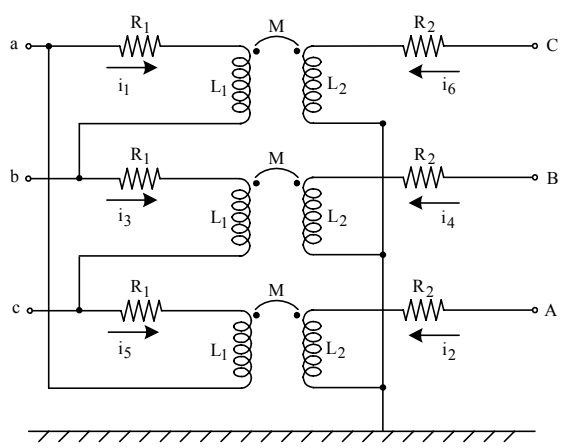

Figura B1. Modelo do circuito acoplado do transformador delta - estrela aterrada (D-Yg).

A matriz $\left[Y_{\text {bus trafo }}\right]$ do transformador elevador mostrada na Tabela 2, para este conexão é detalhada a seguir, onde as três submatrizes $Y_{p p}, Y_{p s}$ e $Y_{s p}$ são singulares, e a submatriz $Y_{s s}$ é não singular. Os taps do lado primário do transformador " $\alpha$ " e os taps do lado secundário " $\beta$ " podem ser diferentes da unidade.

$$
Y_{\text {bus trafo }}=\left[\begin{array}{ccc:ccc}
\frac{2 y_{t}}{3 \alpha^{2}} & -\frac{y_{t}}{3 \alpha^{2}} & -\frac{y_{t}}{3 \alpha^{2}} & -\frac{y_{t}}{\sqrt{3} \alpha \beta} & 0 & \frac{y_{t}}{\sqrt{3} \alpha \beta} \\
-\frac{y_{t}}{3 \alpha^{2}} & \frac{2 y_{t}}{3 \alpha^{2}} & -\frac{y_{t}}{3 \alpha^{2}} & \frac{y_{t}}{\sqrt{3} \alpha \beta} & -\frac{y_{t}}{\sqrt{3} \alpha \beta} & 0 \\
-\frac{y_{t}}{3 \alpha^{2}} & -\frac{y_{t}}{3 \alpha^{2}} & \frac{2 y_{t}}{3 \alpha^{2}} & 0 & \frac{y_{t}}{\sqrt{3} \alpha \beta} & -\frac{y_{t}}{\sqrt{3} \alpha \beta} \\
\hdashline-\frac{y_{t}}{\sqrt{3} \alpha \beta} & \frac{y_{t}}{\sqrt{3} \alpha \beta} & 0 & \frac{y_{t}}{\beta^{2}} & 0 & 0 \\
0 & -\frac{y_{t}}{\sqrt{3} \alpha \beta} & \frac{y_{t}}{\sqrt{3} \alpha \beta} & 0 & \frac{y_{t}}{\beta^{2}} & 0 \\
\frac{y_{t}}{\sqrt{3} \alpha \beta} & 0 & -\frac{y_{t}}{\sqrt{3} \alpha \beta} & 0 & 0 & \frac{y_{t}}{\beta^{2}}
\end{array}\right]
$$

\section{REFERÊNCIAS}

[1] C. S. Cheng, and D. Shirmohammadi, "A three-phase power flow method for real-time distribution system analysis", IEEE Transaction on Power Systems, vol. 10, no. 2, pp. 671-679, May 1995.

[2] R. M. Ciric, A. Padilha-Feltrin, and L. F. Ochoa, "Power flow in fourwire distribution networks-general approach", IEEE Transaction on Power Systems, vol. 18, no. 4, pp. 1283-1290, Nov. 2003.

[3] D. Shirmohammadi, H. W. Hong, A. Semlyen, and G. X. Luo, "A compensation-based power flow method for weakly meshed distribution and transmission networks", IEEE Transaction on Power Systems, vol. 3, no. 2, pp. 753-762, May. 1988.

[4] B. Alencar Souza, H. D. de Mac edo Braz, J. M. Cavalcante de Albuquerque, J. G. Gerlach Gutteres, "Radial Distribution Systems Power Flow with Distributed Generation: Modified Power Summation Method", IEEE LATIN AMERICA TRANSACTIONS, Vol. 4, No. 3, pp. 192-197, May 2006.

[5] Z. Wang, F. Chen, and J. Li, "Implementing transformer noda admittance matrices into backward / forward sweep-based power flow analysis for unbalanced radial distribution systems", IEEE Power Transaction on Power Systems, vol. 19, no. 4, pp. 1831-1836, Nov. 2004.

[6] Peng Xiao, and David C. Yu, "A unified three-phase transformer model for distribution load flow calculations", IEEE Transaction on Powe Systems, vol. 21, no. 1, pp. 153-159, February 2006.

[7] M. S. Chen and W. E. Dillon, "Power system modeling", IEEE Proc., pp. 901-915, July 1974.

[8] J. Arrillaga, C. P. Arnold, and B. J. Harker, "Computer modeling of electrical power systems”, IEEE PES. 2nd ed. New York: Wiley, 2001.

[9] W. H. Kersting, "Distribution system modeling and analysis", Boca Raton, Florida, CRC Pres, 2002.

[10] T. H. Chen, M. S. Chen, T. Inoue, P. Kotas, and E. A. Chebli, "Threephase cogenerator and transformer models for distribution system analysis", IEEE Transaction on Power Delivery, vol. 6, no. 4, pp. 1671681 , Oct. 1991

[11] D. E. Rodas, "Modelagem de transformadores para sistemas de distribuição", ETELEC- UNESP, Ilha Solteira, Aug. 2005.

[12] A. Tan, W. H. E. Liu, and D. Shirmohammadi, "Transformer and load modeling in short circuit analysis for distribution systems", IEEE Transaction on Power Systems, vol 12, no. 3, pp. 1315- 1322, Aug. 1997.

[13] G. X. Luo and A. Semlyen, "Efficient load flow for large weakly meshed networks", IEEE Transactions on Power Systems, vol. 5, no. 4, pp. 1309-1316, Nov. 1990.

[14] W. H. Kersting, "Radial distribution test feeders," IEEE Transactions on Power Systems, vol. 6, no. 3, pp. 975-985, Aug. 1991.

José L. Choque graduado da Universidade Nacional de São Agustín de Arequipa (U.N.S.A.) - Arequipa - Peru, em 2001. Atualmente ele é um estudante de M.Sc. em Sistemas Elétricos de Potência na UNESP - Ilha Solteira, SP, Brazil. Seu principal interesse é analise de sistemas de distribuição.

Dario E. Rodas Rendón Engenheiro Eletricista da Universidade Tecnológica de Pereira (UTP), Colômbia, 1975, Especialista em sistemas de transmissão e distribuição de energia elétrica na Universidade dos Andes, 1989. Atualmente ele é professor na UTP. Sua área de interesse é planejamento em sistemas de energia elétrico, maquinas elétrica.

Antonio Padilha Feltrin (SM'06) cursou engenharia elétrica na EFEI,obteve M.Sc. e Ph.D. da UNICAMP, Brazil. Atualmente, é professor titular da UNESP - Ilha Solteira, SP. De 1995 até 1997, foi prof, visitante no departamento ECE da Universidade de Wisconsin - Madison. Sua área de interesse é em analise e controle de sistemas de potência. 